
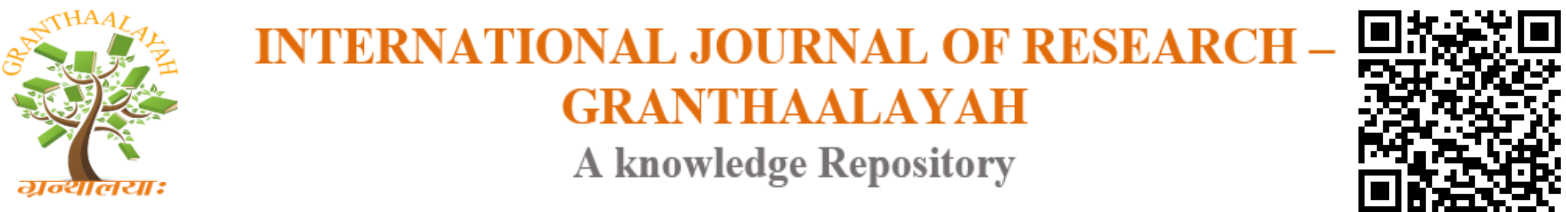

Science

\title{
UNSTEADY MHD HEAT AND MASS TRANSFER FLOW OF A RADIATING FLUID PAST AN ACCELERATED INCLINED POROUS PLATE WITH HALL CURRENT
}

\author{
G. Sivaiah ${ }^{1}$, K. Jayarami Reddy ${ }^{2}$ \\ ${ }^{1}$ Research Scholar, Department of Mathematics, Rayalaseema Univesity, Kurnool-518002, A.P., \\ India \\ ${ }^{2}$ Department of Mathematics, K. L. University, Vaddeswaram, Guntur-522502, A.P. India
}

\begin{abstract}
In this paper an analysis has been performed to study the effects of Hall current and radiation of MHD free convective heat and mass transfer flow of a radiating fluid past an accelerated inclined porous plate with hall current in presence of thermal diffusion and heat source. The solutions for velocity, temperature and concentration distributions are obtained by using Laplace transform technique. The expressions for skin friction, Nusselt number and Sherwood number are also derived. The variations in fluid velocity, temperature and species concentration are shown graphically, whereas numerical values of skin friction, Nusselt number and Sherwood number are presented in tabular form for various values of physical parameters.
\end{abstract}

Keywords: Hall Current; Thermal Radiation; Heat and Mass Transfer; Thermal Diffusion; Accelerated Inclined Porous Plate.

Cite This Article: G. Sivaiah, and K. Jayarami Reddy. (2017). "UNSTEADY MHD HEAT AND MASS TRANSFER FLOW OF A RADIATING FLUID PAST AN ACCELERATED INCLINED POROUS PLATE WITH HALL CURRENT.” International Journal of Research - Granthaalayah, 5(7), 42-59. https://doi.org/10.29121/granthaalayah.v5.i7.2017.2107.

\section{Introduction}

The studies related to boundary layer flow, heat and mass transfer over an inclined plate has generated much interest from astrophysical, renewable energy systems and also hypersonic aerodynamics researchers for a number of decades. There has been a renewed interest in studying MHD flow and heat transfer in porous medium due to the effect of magnetic fields on the boundary layer flow control and on the performance of many systems using electrically conducting fluids. In addition, this type of flow finds applications in many engineering problems such as MHD generators, plasma studies, nuclear reactors, MHD pumps, MHD bearing and geothermal energy extractions. 
In industries, many transport processes exist in which heat and mass transfer takes place simultaneously as a result of combined buoyancy effect of thermal diffusion and diffusion through chemical species. The phenomenon of heat and mass transfer frequently exist in chemically processed industries such as food processing and polymer production. Free convection flow involving coupled heat and mass transfer occurs frequently in nature. For this flow, the driving forces arise due to the temperature and concentration variations in the fluid. For example, in atmospheric flows, thermal convection resulting from heating of the earth by sunlight is affected by differences in water vapor concentration. The study of effects of magnetic field on free convection flow is important in liquid-metals, electrolytes and ionized gases. The combined effects of convective heat and mass transfer on the flow of a viscous, incompressible and electrically conducting fluid has many engineering and geophysical applications such as in geothermal reservoirs, drying of porous solids, thermal insulation, and enhanced oil recovery, cooling of nuclear reactor and underground energy transports. Rapits and Singh [1] studied the effects of uniform transverse magnetic field on the free convection flow of an electrically conducting fluid past an infinite vertical plate for the classes of impulsive and uniformly accelerated motions of the plate. Duwairi and Al-Kablawi [2] formulated and analyzed the MHD conjugative heat transfer problem from vertical surfaces embedded in saturated porous media. Seddeek [3] analyzed the effect of variable viscosity and magnetic field on the flow and heat transfer past a continuously moving porous plate. Abdelkhalek [4] investigated the effects of mass transfer on steady two-dimensional laminar MHD mixed convection flow. Chowdhury and Islam [5] presented a theoretical analysis of a MHD free convection flow of a viso-elastic fluid adjacent to a vertical porous plate. Singh [6] studied heat and mass transfer in mhd boundary layer flow past an inclined plate with viscous dissipation in porous medium. Rajesh and Vijaya kumar verma [7] analyzed radiation and mass transfer effects on MHD free convection flow past an exponentially accelerated vertical plate with variable temperature.

Takhar and Ram [8] have studied MHD free convection flow of water through a porous medium. MHD free convection near a moving vertical plate in the presence of thermal radiation is studied by Das and Das [9]. The effects of variable thermal conductivity and heat source/sink on MHD flow near a stagnation point on a linearly stretching sheet are studied by Sharma and Singh [10]. Chen [11] considered the problem of combined heat and mass transfer of electrically conducting fluid in MHD natural convection adjacent to a vertical surface with ohmic heating. The influence of a uniform transverse magnetic field on the motion of an electrically conducting fluid past a stretching sheet was investigated by Pavlov [12], Chakravarty and Gupta [13], Andersson [14], Andersson et al. [15]. Alam et. al [16] studied the combined effect of viscous dissipation and Joule heating on steady MHD free convective heat and mass transfer flow of a viscous incompressible fluid past a semiinfinite inclined radiate isothermal permeable moving surface in the presence of thermophoresis.

In most cases, the Hall term was ignored in applying Ohm's law as it has no marked effect for small and moderate values of the magnetic field. However, the current trend for the application of MHD is towards a strong magnetic field, so that the influence of electromagnetic force is noticeable [17]. Under these conditions, the Hall current is important and it has a marked effect on the magnitude and direction of the current density and consequently on the magnetic force 
term. Attia [18] has studied the influence of the Hall current on the velocity and temperature fields of an unsteady flow of a conducting Newtonian fluid between two infinite non-conducting horizontal parallel stationary and porous plates. Unsteady MHD fee convection flow past an exponentially accelerated vertical plate with mass transfer, chemical reaction and thermal radiation was examined by Raju et al. [19]. MHD three dimensional Couette flow past a porous plate with heat transfer was considered by Ravikumar et al. [20]. Radiation and mass transfer effects on a free convection flow through a porous medium bounded by a vertical surface were considered by Raju et al. [21]. Unsteady MHD radiative and chemically reactive free convection flow near a moving vertical plate in porous medium was investigated by Reddy et al. [22]. Analytical study was carried out by Raju et al. [23] on MHD free convictive, dissipative boundary layer flow past a porous vertical surface in the presence of thermal radiation, chemical reaction and constant suction. Heat transfer effects were examined by Raju et al. [24] on a viscous dissipative fluid flow past a vertical plate in the presence of induced magnetic field. Thermal and solutal buoyancy effect on MHD boundary layer flow of a visco-elastic fluid past a porous plate with varying suction and heat source in the presence of thermal diffusion was considered by Chandra et al. [25]. Heat and mass transfer in MHD mixed convection flow on a moving inclined porous plate was investigated by Raju et al. [26]. Convective ramped temperature and concentration boundary layer flow of a chemically reactive heat absorbing and radiating fluid over a vertical plate in conducting field with Hall current was considered by Reddy et al. [27]. Magnetohydrodynamic convective double diffusive laminar boundary layer flow past an accelerated vertical plate was considered by Chandra et al. [28]. Hall current effects on unsteady MHD flow in a rotating parallel plate channel bounded by porous bed on the lower half Darcy lap wood model were investigated by Harikrishna et al. [29]. Hall current effects on MHD free convection flow through a porous medium bounded by a vertical surface were also investigated by Reddy et al. [30]. Magneto-convective and radiation absorption fluid flow past an exponentially accelerated vertical porous plate with variable temperature and concentration was investigated by Reddy et al. [31]. Unsteady MHD free convective double diffusive viscoelastic fluid flow past an inclined permeable plate in the presence of viscous dissipation and heat absorption was studied by Umamaheswar et al. [32]. Soret effect due to mixed convection on unsteady magnetohydrodynamic flow past a semi-infinite vertical permeable moving plate in presence of thermal radiation, heat absorption and homogenous chemical reaction was considered by Raju et al. [33]. MHD rotating heat and mass transfer free convective flow past an exponentially accelerated isothermal plate with fluctuating mass diffusion was considered by Phillip et al. [34]. Unsteady MHD free convection flow past an exponentially accelerated vertical plate with mass transfer, chemical reaction and thermal radiation was considered by Chamkha et al. [35]. Numerical study has been carried out by Umamaheswar et al. [36] on magnetoconvective and radiation absorption fluid flow past an exponentially accelerated vertical porous plate with variable temperature and concentration in the presence of Soret and Dufour effects. MHD transient free convection and chemically reactive flow past a porous vertical plate with radiation and temperature gradient dependent heat source in slip flow regime was observed by Rao et al. [37]. Motivated by the above studies in this manuscript an attempt is made to investigate heat and mass transfer characteristics of a Newtonian fluid in the presence of Hall current.

The aim of the present investigation is to analyze the effects of Hall current and radiation of MHD unsteady free convection heat and mass transfer flow of a viscous, electrically conducting 
incompressible fluid near an infinite accelerated inclined plate embedded in porous medium which moves with time dependent velocity under the influence of uniform magnetic field, applied normal to the plate. A general exact solution of the governing partial differential equation is obtained by using Laplace transform technique.

\section{Formulation of the Problem}

Consider unsteady free convection heat and mass transfer flow of a viscous incompressible and electrically conducting fluid along an infinite non-conducting accelerated inclined plate with an acute angle $\alpha$ through a porous medium. $x^{*}$ direction is taken along the leading edge of the inclined plate and $\mathrm{y}^{*}$ is normal to it and extends parallel to $\mathrm{x}^{*}$-axis. A magnetic field of strength $\mathrm{B} 0$ is introduced to the normal to the direction to the flow.

Initially for time $t^{*} \leq 0$, the plate and the fluid are maintained at the same constant temperature $T_{\infty}^{*}$ in a stationary condition with the same species concentration $C_{\infty}^{*}$ at all points. Subsequently $\left(t^{*}>0\right)$, the plate is assumed to be accelerating with a velocity $\mathrm{U}_{0} \mathrm{f}\left(\mathrm{t}^{*}\right)$ in its own plane along the $\mathrm{x}^{*}$-axis, instantaneously the temperature of the plate and the concentration are raised to $\mathrm{T}_{\mathrm{w}}{ }^{*}$ and $\mathrm{C}_{\mathrm{w}}{ }^{*}$ respectively, which are hereafter regarded as constant. The flow of the fluid is assumed to be in the direction of the $\mathrm{x}^{*}$-axis, so the physical quantities are functions of the space coordinate $\mathrm{y}^{*}$ and time $\mathrm{t}^{*}$ only. Taking into consideration the assumption made above, in accordance with the usual Boussinesq approximation, the governing equations for unsteady free convective boundary layer flow of viscous incompressible and electrical conducting fluid along infinite accelerated inclined plate through a porous medium with hall current, radiation and thermal diffusion in two dimensional flow can be expressed as:

Momentum equation:

$$
\frac{\partial u^{*}}{\partial t^{*}}=\vartheta \frac{\partial^{2} u^{*}}{\partial y^{* 2}}+g \beta^{*}\left(T^{*}-T_{\infty}^{*}\right) \cos \alpha+g \beta^{*}\left(C^{*}-C_{\infty}^{*}\right) \cos \alpha-\frac{\vartheta}{K^{*}} u^{*}-\frac{\sigma B_{0}^{2}}{\rho\left(1+m^{2}\right)} u^{*}
$$

Energy Equation:

$$
\frac{\partial T^{*}}{\partial t^{*}}=\frac{K^{*}}{\rho C_{p}} \frac{\partial^{2} T^{*}}{\partial y^{* 2}}-\frac{1}{\rho C_{p}} \frac{\partial q_{r}^{*}}{\partial y^{*}}-Q^{*}\left(T^{*}-T_{\infty}^{*}\right)
$$

Concentration equation:

$$
\frac{\partial C^{*}}{\partial t^{*}}=D_{M} \frac{\partial^{2} C^{*}}{\partial y^{* 2}}-D_{T} \frac{\partial^{2} T^{*}}{\partial y^{* 2}}
$$

where $\mathrm{u}^{*}$ velocity, $\mathrm{T}^{*}$ is the temperature, $\mathrm{C}^{*}$ is the species concentration and $\mathrm{g}$ is the acceleration due to gravity.

The initial and boundary conditions corresponding to the present problem are

$$
\begin{aligned}
& u^{*}\left(y^{*}, t^{*}\right)=0, T^{*}\left(y^{*}, t^{*}\right)=T_{\infty}^{*}, C^{*}\left(y^{*}, t^{*}\right)=C_{\infty}^{*} \text { for } y^{*} \geq 0 \text { and } t^{*} \leq 0 \\
& \left.u^{*}\left(0, t^{*}\right)=u_{0} e^{a_{0}{ }^{*} t^{*}} T^{*}\left(0, t^{*}\right)=T_{w}^{*}, C^{*}\left(0, t^{*}\right)=C_{w}^{*} \text { for } t^{*} \leq 0\right) \\
& u^{*} \rightarrow 0, T^{*} \rightarrow T_{\infty}^{*}, C^{*} \rightarrow C_{\infty}^{*} y * \rightarrow \infty \text { and for } t^{*}>0 \\
& \text { The radiative heat flux } q_{r}^{*} \text { is given by }
\end{aligned}
$$

$\frac{\partial q_{r}^{*}}{\partial y^{*}}=4\left(T^{*}-T_{\infty}^{*}\right) I^{*}$

Where $I^{*}=\int_{0}^{\infty} K_{\lambda w} \frac{\partial e_{b \lambda}}{\partial T^{*}} d \lambda, K_{w \lambda}$ is absorption coefficient and $e_{b \lambda}$ is Plank function. 
To reduce the above equations into non-dimensional form for convenience, let us introduce the following dimensionless variables and parameters:

$$
\begin{aligned}
& u_{0}=\frac{u_{0}^{*}}{U_{0}}, \quad y=\frac{y^{*} U_{0}}{\vartheta}, t=\frac{t^{*} U_{0}^{2}}{\vartheta}, G_{r}=\frac{\vartheta g \beta_{T}\left(T_{W}^{*}-T_{\infty}^{*}\right)}{U_{0}^{3}}, M=\frac{\sigma B_{0}^{2} \vartheta}{\rho U_{0}^{2}}, \\
& P_{r}=\frac{\rho \vartheta C_{p}}{k}, G_{m}=\frac{\vartheta g \beta_{C}\left(C_{w}^{*}-C_{\infty}^{*}\right)}{U_{0}^{3}}, S_{C}=\frac{\vartheta}{D_{M}}, S_{0}=\frac{\left(T_{w}^{*}-T_{\infty}^{*}\right) D_{t}}{\left(C_{w}^{*}-C_{\infty}^{*}\right) \vartheta}, \\
& k=\frac{k^{*} U_{0}^{2}}{\vartheta^{2}}, \gamma=\frac{k_{1}^{*} \vartheta}{U_{0}^{2}}, \omega=\frac{\omega^{*} \vartheta}{U_{0}^{2}}, \quad Q=\frac{Q^{*} \vartheta^{2}}{k * U_{0}^{2}} \\
& \theta=\frac{\left(T^{*}-T_{\infty}^{*}\right)}{T_{w}^{*}-T_{\infty}^{*}}, C=\frac{\left(C^{*}-C_{\infty}^{*}\right)}{C_{w}^{*}-C_{\infty}^{*}}, \quad F=\frac{4 \vartheta I^{*}}{\rho C_{p} U_{0}^{2}}, a_{o}=\frac{a_{0}^{*} \vartheta}{U_{0}^{2}}
\end{aligned}
$$

where $\mathrm{Gr}$ is the thermal Grashof number, Gm is the mass Grashof number, $\mathrm{k}$ is the permeability parameter, $\mathrm{M}$ is the magnetic parameter, $\mathrm{m}$ is the hall current parameter, Pr is Prandtl number, Sc is Schmidt number, $\beta_{\mathrm{T}}$ is thermal expansion coefficient, $\beta_{\mathrm{C}}$ is concentration expansion coefficient $\mathrm{a}_{\mathrm{o}}{ }^{*}$ is dimensional accelerating parameter and other physical variables have their usual meanings.

With the help of (6), the governing equations (1) to (3) reduce to

$$
\begin{aligned}
& \frac{\partial u}{\partial t}=\frac{\partial^{2} u}{\partial y^{2}}+G_{r}(\cos \alpha) \theta+G_{m}(\cos \alpha) C-N u \\
& \frac{\partial^{2} \theta}{\partial y^{2}}-P_{r} \frac{\partial \theta}{\partial t}+(F+Q) \theta=0 \\
& \frac{\partial^{2} C}{\partial y^{2}}-S_{c} \frac{\partial C}{\partial t}+S_{C} S_{0} \frac{\partial^{2} \theta}{\partial y^{2}}=0
\end{aligned}
$$

The corresponding initial and boundary conditions in non-dimensional form are:

$u(y, t)=0, \theta(y, t)=0, C(y, t)=0$ for $y^{*} \geq 0$ and $t^{*} \leq 0$

$u(0, t)=e^{a_{0} t}, \theta(0, t)=1, C(0, t)=1$ for $\left.t^{*}>0\right)$

$u \rightarrow 0, \quad \theta \rightarrow 0, C \rightarrow 0 \quad y \rightarrow \infty$ and for $t>0$

\section{Solution of the Problem}

In order to obtain the analytical solutions of the system of differential equations (7) to (9), we shall use the Laplace transform technique.

Applying the Laplace transform (with respect to time t) to equations (7) to (9) and boundary conditions (10), we get

$$
\begin{gathered}
\bar{\theta}=\frac{1}{s} \exp \left(-y \sqrt{P_{r}} \sqrt{s+S_{1}}\right) \\
\bar{C}=\frac{1}{s} \exp \left(-y \sqrt{S_{c}} \sqrt{s}\right)-m_{2} \frac{1}{s+m_{3}} \exp \left(-y \sqrt{S_{c}} \sqrt{s}\right)-m_{6} \frac{1}{s} \exp \left(-y \sqrt{S_{c}} \sqrt{s}\right) \\
\quad+\frac{m_{6}}{s+m_{5}} \exp \left(-y \sqrt{S_{c}} \sqrt{s}\right)+m_{2} \frac{1}{s+m_{3}} \exp \left(-y \sqrt{P_{r}} \sqrt{s+S_{1}}\right) \\
\quad+m_{6} \frac{1}{s} \exp \left(-y \sqrt{P_{r}} \sqrt{s+S_{1}}\right)-\frac{m_{6}}{s+m_{5}} \exp \left(-y \sqrt{P_{r}} \sqrt{s+S_{1}}\right) \\
\bar{u}(y, s)=\bar{f}(s) \exp (-y \sqrt{s+N})+\frac{m_{29}}{s} \exp (-y \sqrt{s+N})+\frac{m_{10}}{s+m_{9}} \exp (-y \sqrt{s+N}) \\
\quad+\frac{m_{30}}{s-m_{12}} \exp (-y \sqrt{s+N})+\frac{m_{31}}{s+m_{3}} \exp (-y \sqrt{s+N})+\frac{m_{32}}{s+m_{5}} \exp (-y \sqrt{s+N}) \\
\quad+\frac{m_{33}}{s+m_{21}} \exp (-y \sqrt{s+N})+\frac{m_{34}}{s} \exp \left(-y \sqrt{P_{r}} \sqrt{s+S_{1}}\right)
\end{gathered}
$$




$$
\begin{aligned}
& -\frac{m_{10}}{s+m_{9}} \exp \left(-y \sqrt{P_{r}} \sqrt{s+S_{1}}\right) \\
& -m_{13} \frac{1}{s} \exp \left(-y \sqrt{S_{c}} \sqrt{s}\right)+\frac{m_{35}}{s-m_{12}} \exp \left(-y \sqrt{S_{c}} \sqrt{s}\right) \\
& \frac{m_{15}}{s+m_{3}} \exp \left(-y \sqrt{S_{c}} \sqrt{s}\right)+\frac{m_{18}}{s-m_{12}} \exp \left(-y \sqrt{S_{c}} \sqrt{s}\right)+\frac{m_{36}}{s+m_{21}} \exp \left(-y \sqrt{P_{r}} \sqrt{s+S_{1}}\right) \\
& +\frac{m_{19}}{s+m_{5}} \exp \left(-y \sqrt{S_{c}} \sqrt{s}\right)+\frac{m_{23}}{s+m_{3}} \exp \left(-y \sqrt{P_{r}} \sqrt{s+S_{1}}\right) \\
& +\frac{m_{28}}{s+m_{5}} \exp \left(-y \sqrt{P_{r}} \sqrt{s+S_{1}}\right)
\end{aligned}
$$

Then, inverting equations (11) to (13) in the usual way we get the general solution of the problem for the temperature $\theta(y, t)$, the species concentration $\mathrm{C}(\mathrm{y}, \mathrm{t})$ and velocity $\mathrm{u}(\mathrm{y}, \mathrm{t})$ for $\mathrm{t}>$ 0 in the non dimensional form as

$$
\begin{aligned}
& \theta=\frac{1}{2}\left[e^{-y \sqrt{P_{r} m_{1}}} \operatorname{erfc}\left(\frac{y \sqrt{P_{r}}}{2 \sqrt{t}}-\sqrt{m_{1} t}\right)+e^{y \sqrt{P_{r} m_{1}}} \operatorname{erfc}\left(\frac{y \sqrt{P_{r}}}{2 \sqrt{t}}+\sqrt{m_{1} t}\right)\right] \\
& C=\operatorname{erfc}\left(\frac{y \sqrt{S_{c}}}{2 \sqrt{t}}\right)-m_{2}\left\{\frac { e ^ { - m _ { 3 } t } } { 2 } \left[e^{-y \sqrt{s_{c}} \sqrt{-m_{3}}} \operatorname{erfc}\left(\frac{y \sqrt{S_{c}}}{2 \sqrt{t}}-\sqrt{-m_{3} t}\right)\right.\right. \\
& \left.\left.+e^{y \sqrt{S_{c}} \sqrt{-m_{3}}} \operatorname{erfc}\left(\frac{y \sqrt{S_{c}}}{2 \sqrt{t}}+\sqrt{-m_{3} t}\right)\right]\right\}-m_{6} \operatorname{erfc}\left(\frac{y \sqrt{S_{c}}}{2 \sqrt{t}}\right) \\
& +m_{6}\left\{\frac { e ^ { - m _ { 5 } t } } { 2 } \left[e^{-y \sqrt{S_{c}} \sqrt{-m_{5}}} \operatorname{erfc}\left(\frac{y \sqrt{S_{c}}}{2 \sqrt{t}}-\sqrt{-m_{5} t}\right)+e^{y \sqrt{s_{c}} \sqrt{-m_{5}}} \operatorname{erfc}\left(\frac{y \sqrt{S_{c}}}{2 \sqrt{t}}+\right.\right.\right. \\
& \left.\left.\left.\sqrt{-m_{5} t}\right)\right]\right\} \quad+m_{2}\left\{\frac{e^{-m_{3} t}}{2}\left[e^{-y \sqrt{P_{r}} \sqrt{-m_{3}+m_{1}}} \operatorname{erfc}\left(\frac{y \sqrt{P_{r}}}{2 \sqrt{t}}-\sqrt{\left(-m_{3}+m_{1}\right) t}\right)\right]\right. \\
& \left.+e^{y \sqrt{P_{r}} \sqrt{-m_{3}+m_{1}}} \operatorname{erfc}\left(\frac{y \sqrt{P_{r}}}{2 \sqrt{t}}+\sqrt{\left(-m_{3}+m_{1}\right) t}\right)\right\} \\
& +m_{6}\left\{\frac{1}{2}\left[e^{-y \sqrt{P_{r}} \sqrt{m_{1}}} \operatorname{erfc}\left(\frac{y \sqrt{P_{r}}}{2 \sqrt{t}}-\left(\sqrt{m_{1} t}\right)\right)+e^{y \sqrt{P_{r}} \sqrt{m_{1}}} \operatorname{erfc}\left(\frac{y \sqrt{P_{r}}}{2 \sqrt{t}}+\left(\sqrt{m_{1} t}\right)\right)\right]\right\} \\
& -m_{6}\left\{\frac { e ^ { - m _ { 5 } t } } { 2 } \left[e^{-y \sqrt{P_{r}} \sqrt{-m_{5}+m_{1}}} \operatorname{erfc}\left(\frac{y \sqrt{P_{r}}}{2 \sqrt{t}}-\sqrt{\left(-m_{5}+m_{1}\right) t}\right)\right.\right. \\
& \left.\left.+e^{y \sqrt{P_{r}} \sqrt{-m_{5}+m_{1}}} \operatorname{erfc}\left(\frac{y \sqrt{P_{r}}}{2 \sqrt{t}}+\sqrt{\left(-m_{5}+m_{1}\right) t}\right)\right]\right\} \\
& u(y, t)=\frac{e^{a_{0} t}}{2}\left[e^{-y \sqrt{a_{0}+N}} \operatorname{erfc}\left(\frac{y}{2 \sqrt{t}}-\sqrt{\left(a_{0}+N\right) t}\right)+e^{y \sqrt{a_{0}+N}} \operatorname{erfc}\left(\frac{y}{2 \sqrt{t}}+\sqrt{\left(a_{0}+N\right) t}\right)\right] \\
& +\emptyset(y, t) \\
& \text { Where } \varnothing(y, t)=\frac{m_{29}}{2}\left[e^{-y \sqrt{N}} \operatorname{erfc}\left(\frac{y}{2 \sqrt{t}}-\sqrt{N t}\right)+e^{y \sqrt{N}} \operatorname{erfc}\left(\frac{y}{2 \sqrt{t}}+\sqrt{N t}\right)\right] \\
& +m_{10} \frac{e^{-m_{9} t}}{2}\left[e^{-y \sqrt{-m_{9}+N}} \operatorname{erfc}\left(\frac{y}{2 \sqrt{t}}-\sqrt{\left(-m_{9}+N\right) t}\right)+\right. \\
& \left.e^{y \sqrt{-m_{9}+N}} \operatorname{erfc}\left(\frac{y}{2 \sqrt{t}}+\sqrt{\left(-m_{9}+N\right) t}\right)\right] \\
& +m_{30} \frac{e^{m_{12} t}}{2}\left[e^{-y \sqrt{m_{12}+N}} \operatorname{erfc}\left(\frac{y}{2 \sqrt{t}}-\sqrt{\left(m_{12}+N\right) t}\right)+\right. \\
& \left.e^{y \sqrt{m_{12}+N}} \operatorname{erfc}\left(\frac{y}{2 \sqrt{t}}+\sqrt{\left(m_{12}+N\right) t}\right)\right] \\
& +m_{31} \frac{e^{-m_{3} t}}{2}\left[e^{-y \sqrt{-m_{3}+N}} \operatorname{erfc}\left(\frac{y}{2 \sqrt{t}}-\sqrt{\left(-m_{3}+N\right) t}\right)+\right. \\
& \left.e^{y \sqrt{-m_{3}+N}} \operatorname{erfc}\left(\frac{y}{2 \sqrt{t}}+\sqrt{\left(-m_{3}+N\right) t}\right)\right] \\
& +m_{32} \frac{e^{-m_{5} t}}{2}\left[e^{-y \sqrt{-m_{5}+N}} \operatorname{erfc}\left(\frac{y}{2 \sqrt{t}}-\sqrt{\left(-m_{5}+N\right) t}\right)+\right. \\
& \left.e^{y \sqrt{-m_{5}+N}} \operatorname{erfc}\left(\frac{y}{2 \sqrt{t}}+\sqrt{\left(-m_{5}+N\right) t}\right)\right]
\end{aligned}
$$




$$
\begin{aligned}
& +m_{33} \frac{e^{-m_{21} t}}{2}\left[e^{-y \sqrt{-m_{21}+N}} \operatorname{erfc}\left(\frac{y}{2 \sqrt{t}}-\sqrt{\left(-m_{21}+N\right) t}\right)+\right. \\
& \left.e^{y \sqrt{-m_{21}+N}} \operatorname{erfc}\left(\frac{y}{2 \sqrt{t}}+\sqrt{\left(-m_{21}+N\right) t}\right)\right] \\
& +\frac{m_{34}}{2}\left[e^{-y \sqrt{P_{r}} \sqrt{m_{1}}} \operatorname{erfc}\left(\frac{y \sqrt{P_{r}}}{2 \sqrt{t}}-\left(\sqrt{m_{1} t}\right)\right)+e^{y \sqrt{P_{r}} \sqrt{m_{1}}} \operatorname{erfc}\left(\frac{y \sqrt{P_{r}}}{2 \sqrt{t}}+\left(\sqrt{m_{1} t}\right)\right)\right] \\
& -m_{10}\left\{\frac { e ^ { - m _ { 9 } t } } { 2 } \left[e^{-y \sqrt{P_{r}} \sqrt{-m_{9}+m_{1}}} \operatorname{erfc}\left(\frac{y \sqrt{P_{r}}}{2 \sqrt{t}}-\sqrt{\left(-m_{9}+m_{1}\right) t}\right)\right.\right. \\
& \left.\left.+e^{y \sqrt{P_{r}} \sqrt{-m_{9}+m_{1}}} \operatorname{erfc}\left(\frac{y \sqrt{P_{r}}}{2 \sqrt{t}}+\sqrt{\left(-m_{9}+m_{1}\right) t}\right)\right]\right\}-m_{13} \operatorname{erfc}\left(\frac{y \sqrt{S_{c}}}{2 \sqrt{t}}\right) \\
& +m_{15}\left\{\frac { e ^ { - m _ { 3 } t } } { 2 } \left[e^{-y \sqrt{-S_{c}} \sqrt{m_{3}}} \operatorname{erfc}\left(\frac{y \sqrt{s_{c}}}{2 \sqrt{t}}-\sqrt{-m_{3} t}\right)+e^{y \sqrt{-S_{c}} \sqrt{m_{3}}} \operatorname{erfc}\left(\frac{y \sqrt{s_{c}}}{2 \sqrt{t}}+\right.\right.\right. \\
& \left.\left.\left.\sqrt{-m_{3} t}\right)\right]\right\} \\
& +m_{18}\left\{\frac{e^{m_{12} t}}{2}\left[e^{-y \sqrt{S_{c}} \sqrt{m_{12}}} \operatorname{erfc}\left(\frac{y \sqrt{S_{c}}}{2 \sqrt{t}}-\sqrt{m_{12} t}\right)+e^{y \sqrt{S_{c}} \sqrt{m_{12}}} \operatorname{erfc}\left(\frac{y \sqrt{S_{c}}}{2 \sqrt{t}}+\sqrt{m_{12} t}\right)\right]\right\} \\
& +m_{19}\left\{\frac{e^{-m_{5} t}}{2}\left[e^{-y \sqrt{S_{c}} \sqrt{m_{5}}} \operatorname{erfc}\left(\frac{y \sqrt{S_{c}}}{2 \sqrt{t}}-\sqrt{-m_{5} t}\right)+e^{y \sqrt{-S_{c}} \sqrt{m_{5}}} \operatorname{erfc}\left(\frac{y \sqrt{S_{c}}}{2 \sqrt{t}}+\sqrt{-m_{5} t}\right)\right]\right\} \\
& +m_{35}\left\{\frac{e^{m_{12} t}}{2}\left[e^{-y \sqrt{S_{c}} \sqrt{m_{12}}} \operatorname{erfc}\left(\frac{y \sqrt{S_{c}}}{2 \sqrt{t}}-\sqrt{m_{12} t}\right)+e^{y \sqrt{S_{c}} \sqrt{m_{12}}} \operatorname{erfc}\left(\frac{y \sqrt{S_{c}}}{2 \sqrt{t}}+\sqrt{m_{12} t}\right)\right]\right\} \\
& +m_{36}\left\{\frac { e ^ { - m _ { 2 1 } t } } { 2 } \left[e^{-y \sqrt{P_{r}} \sqrt{-m_{21}+m_{1}}} \operatorname{erfc}\left(\frac{y \sqrt{P_{r}}}{2 \sqrt{t}}-\sqrt{\left(-m_{21}+m_{1}\right) t}\right)\right.\right. \\
& \left.\left.+e^{y \sqrt{P_{r}} \sqrt{-m_{21}+m_{1}}} \operatorname{erfc}\left(\frac{y \sqrt{P_{r}}}{2 \sqrt{t}}+\sqrt{\left(-m_{21}+m_{1}\right) t}\right)\right]\right\} \\
& +m_{23}\left\{\frac { e ^ { - m _ { 3 } t } } { 2 } \left[e^{-y \sqrt{P_{r}} \sqrt{-m_{3}+m_{1}}} \operatorname{erfc}\left(\frac{y \sqrt{P_{r}}}{2 \sqrt{t}}-\sqrt{\left(-m_{3}+m_{1}\right) t}\right)\right.\right. \\
& \left.\left.+e^{y \sqrt{P_{r}} \sqrt{-m_{3}+m_{1}}} \operatorname{erfc}\left(\frac{y \sqrt{P_{r}}}{2 \sqrt{t}}+\sqrt{\left(-m_{3}+m_{1}\right) t}\right)\right]\right\} \\
& +m_{28}\left\{\frac { e ^ { - m _ { 5 } t } } { 2 } \left[e^{-y \sqrt{P_{r}} \sqrt{-m_{5}+m_{1}}} \operatorname{erfc}\left(\frac{y \sqrt{P_{r}}}{2 \sqrt{t}}-\sqrt{\left(-m_{5}+m_{1}\right) t}\right)\right.\right. \\
& \left.\left.+e^{y \sqrt{P_{r}} \sqrt{-m_{5}+m_{1}}} \operatorname{erfc}\left(\frac{y \sqrt{P_{r}}}{2 \sqrt{t}}+\sqrt{\left(-m_{5}+m_{1}\right) t}\right)\right]\right\}
\end{aligned}
$$

\section{Skin-friction}

The quantities of physical interest are the skin-friction due to velocity is given by

$$
\begin{aligned}
& \boldsymbol{\tau}=\left(\frac{\partial u}{\partial y}\right)_{\boldsymbol{y}=\mathbf{0}} \\
& \quad=\frac{1}{2}\left[\frac{-2}{\sqrt{\pi \mathrm{t}}} \exp \left(-\left(a_{0}+N\right) t\right)+\sqrt{a_{0}+N}\left[\operatorname{erfc}\left(\sqrt{\left(a_{0}+N\right) t}\right)-\operatorname{erfc}\left(-\sqrt{\left(a_{0}+N\right) t}\right)\right]\right]+ \\
& \left(\frac{\partial \emptyset}{\partial y}\right)_{y=0}
\end{aligned}
$$

\section{Where}

$\left(\frac{\partial \emptyset}{\partial \boldsymbol{y}}\right)_{\boldsymbol{y}=\mathbf{0}}=\frac{m_{29}}{2}\left[\frac{-2}{\sqrt{\pi t}} e^{-N t}+\sqrt{N}(\operatorname{erfc}(\sqrt{N t})-\operatorname{erfc}(-\sqrt{N t}))\right]$

$+\frac{m_{10}}{2} e^{-m_{9} t}\left[\frac{-2}{\sqrt{\pi \mathrm{t}}} e^{-\left(N-m_{9}\right) t}+\sqrt{\left(N-m_{9}\right)}\left(\operatorname{erfc}\left(\sqrt{\left(N-m_{9}\right) t}\right)-\operatorname{erfc}\left(-\sqrt{\left(N-m_{9}\right) t}\right)\right)\right]$ 
$+\frac{m_{30}}{2} e^{-m_{12} t}\left[\frac{-2}{\sqrt{\pi \mathrm{t}}} e^{-\left(N+m_{12}\right) t}+\right.$

$\left.\sqrt{\left(N+m_{12}\right)}\left(\operatorname{erfc}\left(\sqrt{\left(N+m_{12}\right) t}\right)-\operatorname{erfc}\left(-\sqrt{\left(N+m_{12}\right) t}\right)\right)\right]$

$+\frac{m_{31}}{2} e^{-m_{3} t}\left[\frac{-2}{\sqrt{\pi \mathrm{t}}} e^{-\left(N-m_{3}\right) t}+\sqrt{\left(N-m_{3}\right)}\left(\operatorname{erfc}\left(\sqrt{\left(N-m_{3}\right) t}\right)-\operatorname{erfc}\left(-\sqrt{\left(N-m_{3}\right) t}\right)\right)\right]$

$+\frac{m_{32}}{2} e^{-m_{5} t}\left[\frac{-2}{\sqrt{\pi \mathrm{t}}} e^{-\left(N-m_{5}\right) t}+\sqrt{\left(N-m_{5}\right)}\left(\operatorname{erfc}\left(\sqrt{\left(N-m_{5}\right) t}\right)-\right.\right.$

$\left.\left.\operatorname{erfc}\left(-\sqrt{\left(N-m_{5}\right) t}\right)\right)\right]$

$+\frac{m_{33}}{2} e^{-m_{21} t}\left[\frac{-2}{\sqrt{\pi \mathrm{t}}} e^{-\left(N-m_{21}\right) t}+\right.$

$\sqrt{\left(N-m_{21}\right)}\left(\operatorname{erfc}\left(\sqrt{\left(N-m_{21}\right) t}\right)-\right.$

$\left.\left.\operatorname{erfc}\left(-\sqrt{\left(N-m_{21}\right) t}\right)\right)\right]$

$+\frac{m_{34}}{2}\left[\frac{-2}{\sqrt{\pi \mathrm{t}}} e^{-m_{1} t}+\sqrt{\left(P_{r} m_{1}\right)}\left(\operatorname{erfc}\left(\sqrt{m_{1} t}\right)-\right.\right.$

$\left.\left.\operatorname{erfc}\left(-\sqrt{m_{1} t}\right)\right)\right]$

$+\frac{m_{10}}{2} e^{-m_{9} t}\left[\frac{-2}{\sqrt{\pi \mathrm{t}}} e^{-\left(m_{1}-m_{9}\right) t}+\right.$

$\left.\sqrt{\left(m_{1}-m_{9}\right) P_{r}}\left(\operatorname{erfc}\left(\sqrt{\left(m_{1}-m_{9}\right) t}\right)-\operatorname{erfc}\left(-\sqrt{\left(m_{1}-m_{9}\right) t}\right)\right)\right]+m_{13}\left[\frac{\sqrt{s_{c}}}{\sqrt{\pi t}}\right]+$

$\frac{m_{35}}{2} e^{-m_{12} t}\left[\frac{-2 \sqrt{S_{c}}}{\sqrt{\pi \mathrm{t}}} e^{-m_{12} t}+\right.$

$\sqrt{\left(S_{c} m_{12}\right)}\left(\operatorname{erfc}\left(\sqrt{m_{12} t}\right)-\operatorname{erfc}\left(-\sqrt{m_{12} t}\right)\right)$

$+\frac{m_{15}}{2} e^{-m_{3} t}\left[\frac{-2 \sqrt{S_{c}}}{\sqrt{\pi \mathrm{t}}} e^{m_{3} t}+\right.$

$\left.\sqrt{\left(-m_{3} S_{c}\right)}\left(\operatorname{erfc}\left(\sqrt{-m_{3} t}\right)-\operatorname{erfc}\left(-\sqrt{-m_{3} t}\right)\right)\right]$

$+\frac{m_{18}}{2} e^{m_{12} t}\left[\frac{-2 \sqrt{S_{c}}}{\sqrt{\pi \mathrm{t}}} e^{-m_{12} t}+\sqrt{\left(m_{12} S_{c}\right)}\left(\operatorname{erfc}\left(\sqrt{m_{12} t}\right)-\operatorname{erfc}\left(-\sqrt{m_{12} t}\right)\right)\right]$

$+\frac{m_{19}}{2} e^{-m_{5} t}\left[\frac{-2 \sqrt{S_{c}}}{\sqrt{\pi \mathrm{t}}} e^{m_{5} t}+\sqrt{\left(-m_{5} S_{c}\right)}\left(\operatorname{erfc}\left(\sqrt{\left(-m_{5}\right) t}\right)-\operatorname{erfc}\left(-\sqrt{\left(-m_{5}\right) t}\right)\right)\right]$

$+\frac{m_{36}}{2} e^{-m_{21} t}$

$\left[\frac{-2 P_{r}}{\sqrt{\pi \mathrm{t}}} e^{-\left(m_{1}-m_{25}\right) t}+\sqrt{\left(m_{1}-m_{25}\right) P_{r}}\left(\operatorname{erfc}\left(\sqrt{\left(m_{1}-m_{25}\right) t}\right)-\right.\right.$

$\left.\left.\operatorname{erfc}\left(-\sqrt{\left(m_{1}-m_{25}\right) t}\right)\right)\right]$

$+\frac{m_{23}}{2} e^{-m_{3} t}$

$\left[\frac{-2 P_{r}}{\sqrt{\pi \mathrm{t}}} e^{-\left(m_{1}-m_{3}\right) t}+\sqrt{\left(m_{1}-m_{3}\right) P_{r}}\left(\operatorname{erfc}\left(\sqrt{\left(m_{1}-m_{3}\right) t}\right)-\right.\right.$

$\left.\left.\operatorname{erfc}\left(-\sqrt{\left(m_{1}-m_{3}\right) t}\right)\right)\right]$

$+\frac{m_{28}}{2} e^{-m_{5} t}$

$\left[\frac{-2 P_{r}}{\sqrt{\pi \mathrm{t}}} e^{-\left(m_{1}-m_{5}\right) t}+\sqrt{\left(m_{1}-m_{5}\right) P_{r}}\left(\operatorname{erfc}\left(\sqrt{\left(m_{1}-m_{5}\right) t}\right)-\right.\right.$

$\left.\left.\operatorname{erfc}\left(-\sqrt{\left(m_{1}-m_{5}\right) t}\right)\right)\right]$

\section{Nusselt number}

An important phenomenon in this study is to understand the effects of $t, P_{r}$ on the Nusselt number. In non dimensional form, the rate of heat transfer is given by

$$
N_{u}=-\left(\frac{\partial \theta}{\partial y}\right)_{y=0}=\frac{1}{2}\left[\frac{-2}{\sqrt{\pi t}} e^{-m_{1} t}+\sqrt{\left(P_{r} m_{1}\right)}\left(\operatorname{erfc}\left(\sqrt{m_{1} t}\right)-\operatorname{erfc}\left(-\sqrt{m_{1} t}\right)\right)\right]
$$




\section{Sherwood Number}

Another important physical quantities of interest is the Sherwood number which in nondimensional form is

$$
\begin{aligned}
& S_{h}=-\left(\frac{\partial C}{\partial y}\right)_{y=0} \\
&=- \quad \sqrt{\frac{S_{c}}{\pi t}}-\frac{m_{2}}{2} e^{m_{3} t}\left[\frac{-2}{\sqrt{\pi \mathrm{t}}} e^{m_{3} t}+\sqrt{\left(-m_{3} S_{c}\right)}\left(\operatorname{erfc}\left(\sqrt{-m_{3} t}\right)-\operatorname{erfc}\left(-\sqrt{-m_{3} t}\right)\right)\right] \\
&+m_{6}\left[\frac{\sqrt{S_{c}}}{\sqrt{\pi \mathrm{t}}}\right] \\
&-\frac{m_{6}}{2} e^{-m_{5} t}\left[\frac{-2}{\sqrt{\pi \mathrm{t}}} e^{m_{3} t}+\sqrt{\left(-m_{5} S_{c}\right)}\left(\operatorname{erfc}\left(\sqrt{-m_{5} t}\right)-\operatorname{erfc}\left(-\sqrt{-m_{5} t}\right)\right)\right] \\
& \quad+\frac{m_{2}}{2} e^{-m_{3} t} \\
& \operatorname{erfc}(-\left.\left.\left.\sqrt{\left(m_{1}-m_{3}\right) t}\right)\right)\right] \\
&+\frac{m_{6}}{2}\left[\frac{-2}{\sqrt{\pi \mathrm{t}}} e^{-\left(m_{1}-m_{3}\right) t}+\sqrt{\left(m_{1}-m_{3}\right) S c}\left(\operatorname{erfc}\left(\sqrt{\left(m_{1}-m_{3}\right) t}\right)-\right.\right. \\
& \quad-\frac{m_{6}}{2} e^{-m_{5} t} \\
& {\left[\frac{-2}{\sqrt{\pi \mathrm{t}}} e^{-\left(m_{1}-m_{5}\right) t}+\sqrt{\left(P_{1} m_{1}\right)}\left(\operatorname{erfc}\left(\sqrt{m_{1} t}\right)-\operatorname{erfc}\left(-\sqrt{m_{1} t}\right)\right)\right] }
\end{aligned}
$$

\section{Results and Discussion}

The system of transformed differential equations (7) - (9) subject to the boundary conditions (10) is solved using Laplace transform technique. To understand the physical meaning of the problem, we have computed the expression for velocity $(u)$, temperature profile $(\theta)$, Concentration $(C)$, Skin friction $(\tau)$, rate of heat transfer in the form of Nussselt Number and rate of mass transfer in the form of Sherwood number for different values of Prandtl number Pr, magnetic field parameter M, Hall current parameter (m), Grashof number (Gr), modified Grashof number $(\mathrm{Gm})$, Schmidt number $(\mathrm{Sc})$, permeability parameter $(\mathrm{k})$, Soret number $\left(\mathrm{S}_{0}\right)$, radiation parameter(F), Heat source parameter (Q), and inclination angle $\alpha$. The consequences of relevant parameters on the flow field are broken down and discuss with the help of graphs of the velocity profiles, temperature profiles, concentration profiles and tables of Skin-friction coefficient, Nusselt number and Sherwood number.

The velocity profiles for different values of physical parameters are presented in figures 1-9. From this it is observed that velocity increases as Pr, F, So, M, Gr, Gm, and Q increase respectively, while the velocity decreases as $\mathrm{Sc}, \alpha$ and $\mathrm{m}$ increase. The temperature profile for different values of Prandtl number Pr and radiation parameter F are shown in figures $10 \& 11$. It is observed $\operatorname{Pr} \& \mathrm{~F}$ increase as temperature decreases. Figure 12 shows the temperature profile for different values of Heat source parameter Q. It is observed that the temperature decreases with the increase of Q. Figures 13 - 16 depict the variation of Concentration filed $\mathrm{C}$ against Schmidt number Sc, Soret number $S_{0}$, radiation parameter F, Heat source parameter Q . It is noticed that the concentration increases with increase in $\mathrm{F}$ and So respectively, while the concentration decreases as Sc and Q increase. 


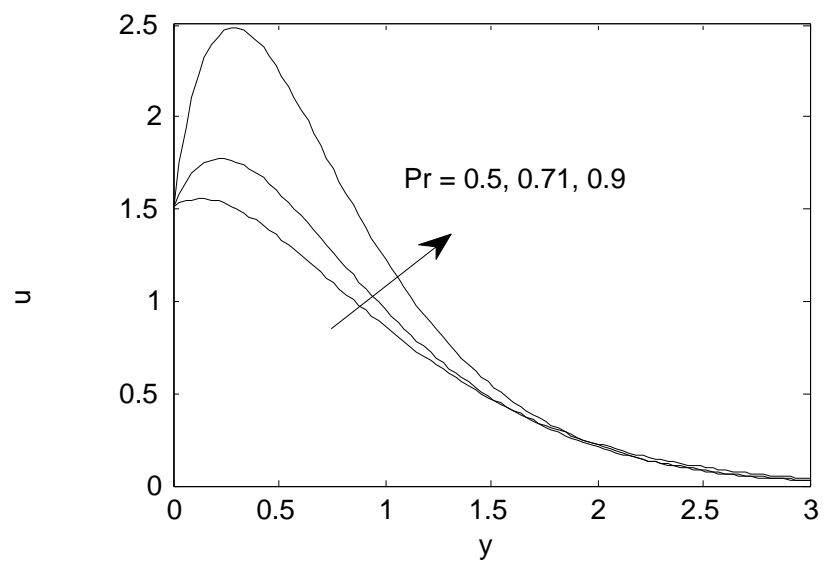

Figure 1: Velocity distribution for various values of Pr

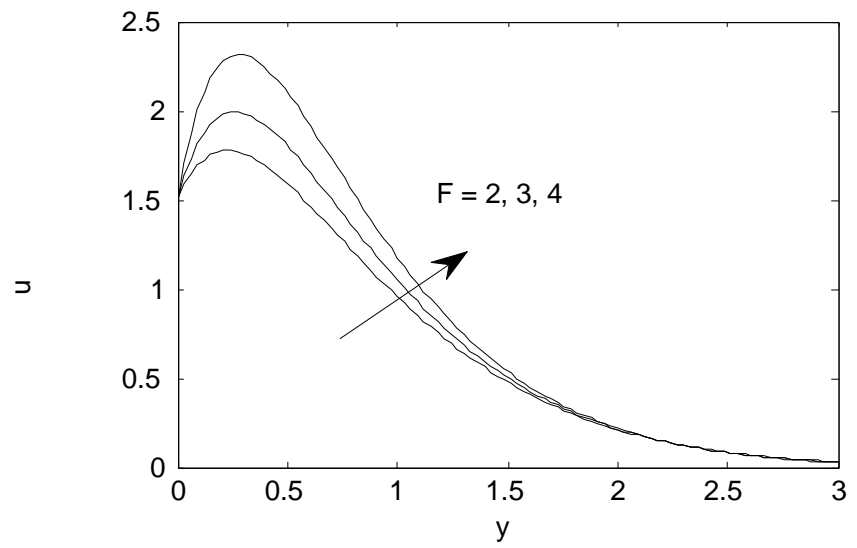

Figure 2: Velocity distribution for various values of $\mathrm{F}$

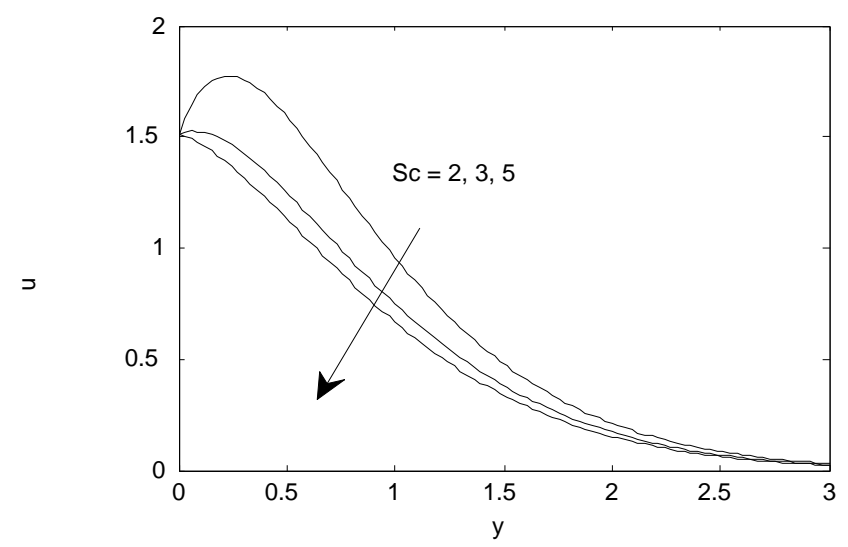

Figure 3: Velocity distribution for various values of Sc 


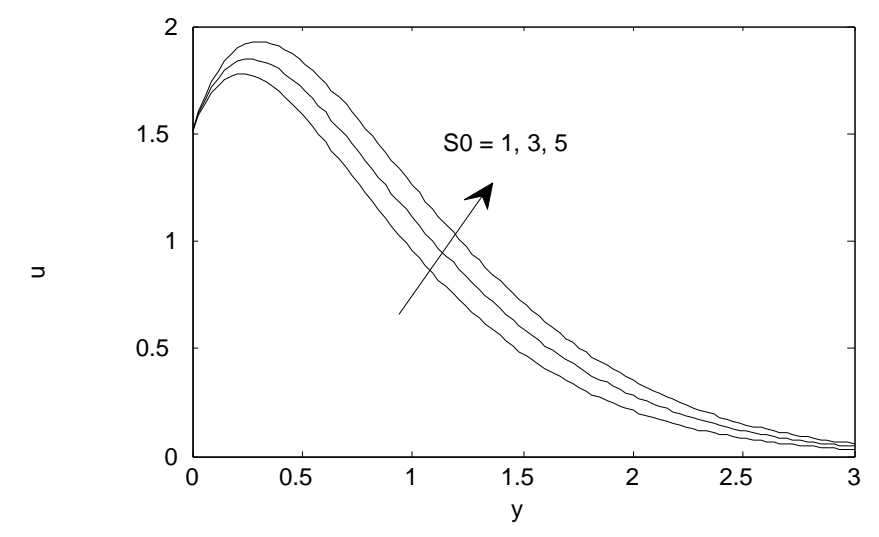

Figure 4: Velocity distribution for various values of S0

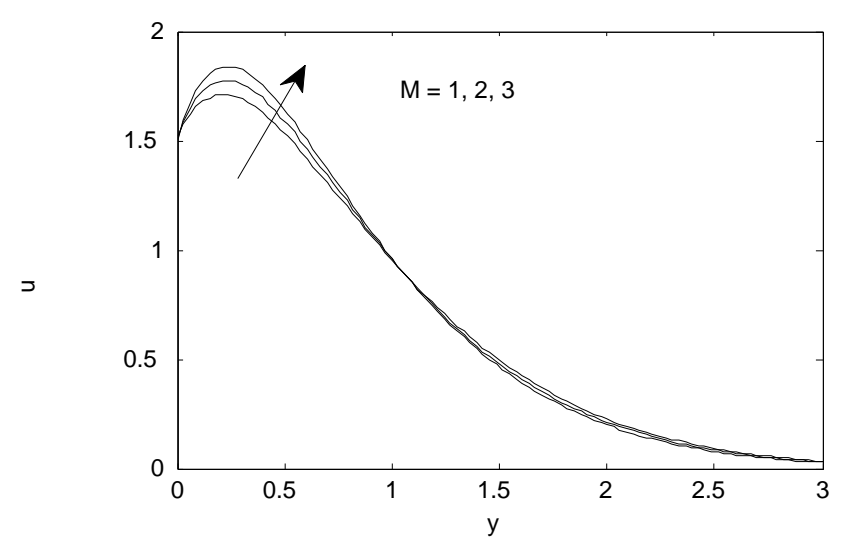

Figure 5: Velocity distribution for various values of $\mathrm{M}$

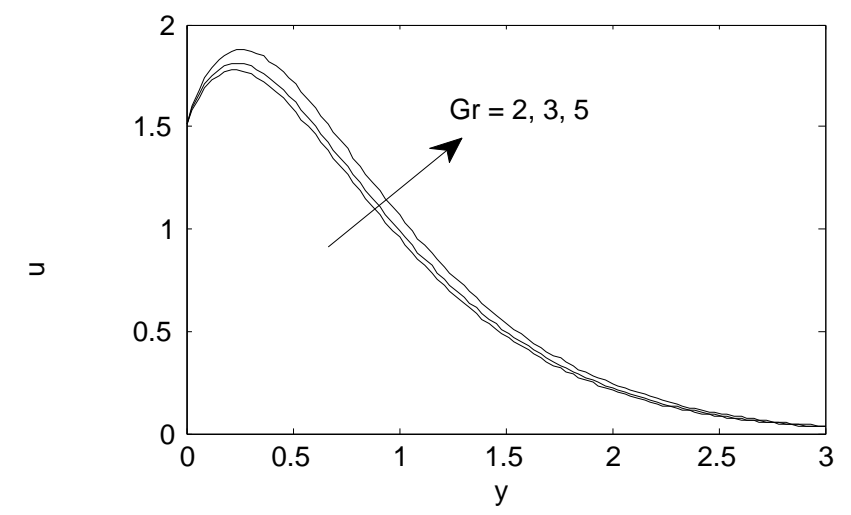

Figure 6: Velocity distribution for various values of $\mathrm{Gr}$ 


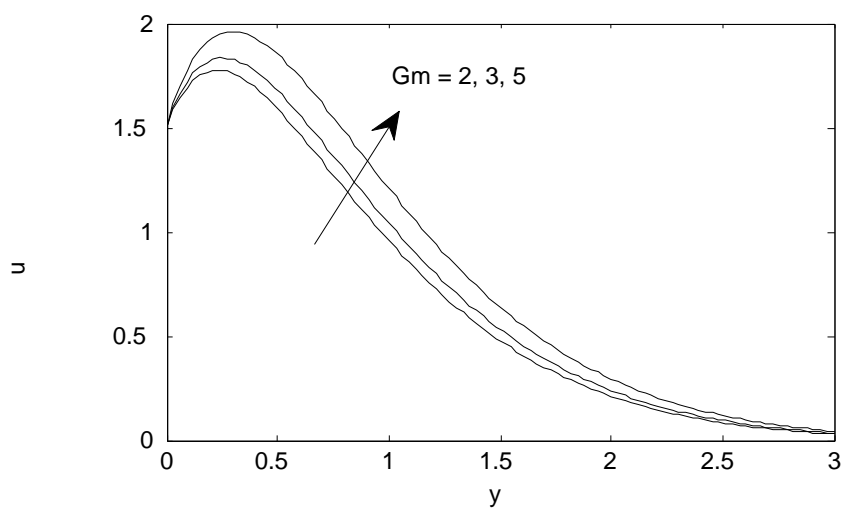

Figure 7: Velocity distribution for various values of $\mathrm{Gm}$

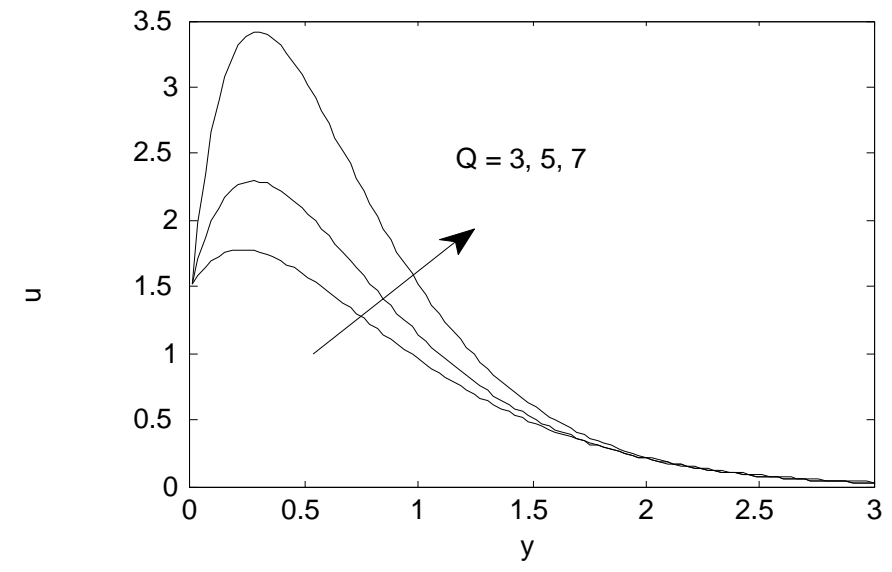

Figure 8: Velocity distribution for various values of $\mathrm{Q}$

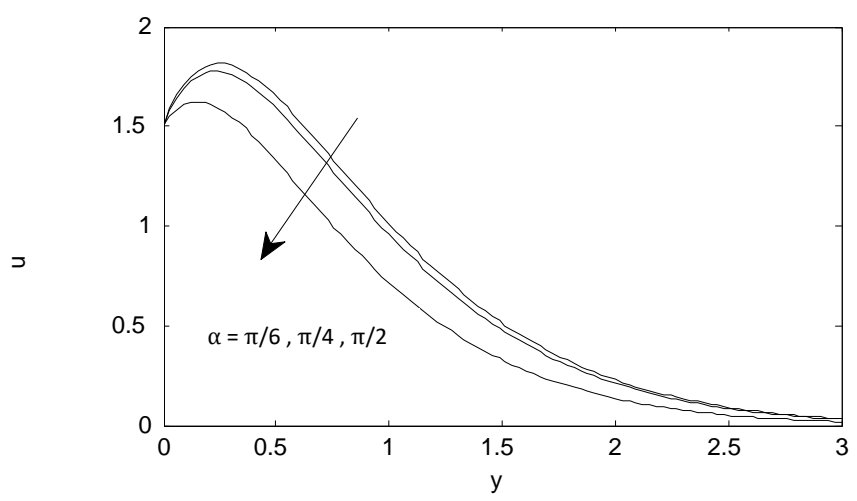

Figure 9: Velocity distribution for various values of Alpha 


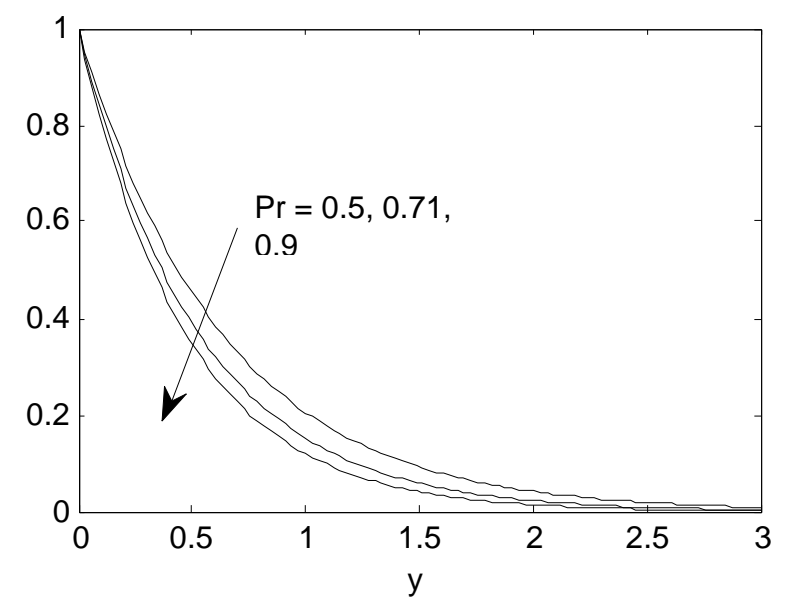

Figure 10: Temperature distribution for various values of $\mathrm{Pr}$

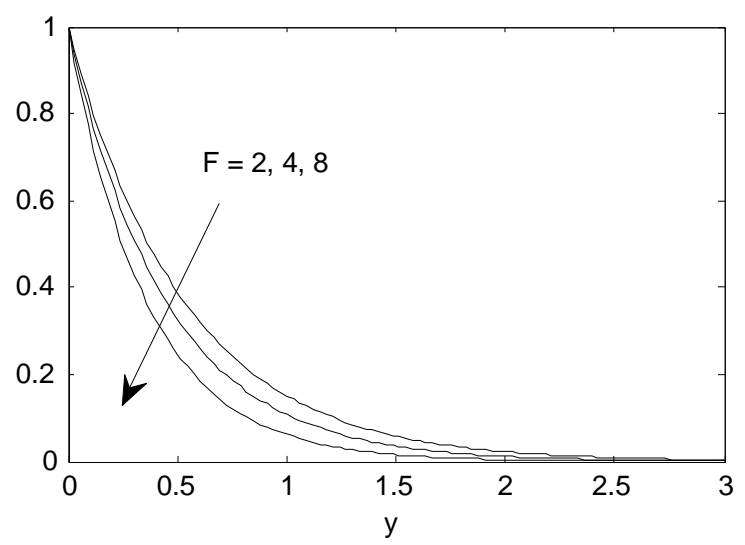

Figure 11: Temperature distribution for various values of $F$

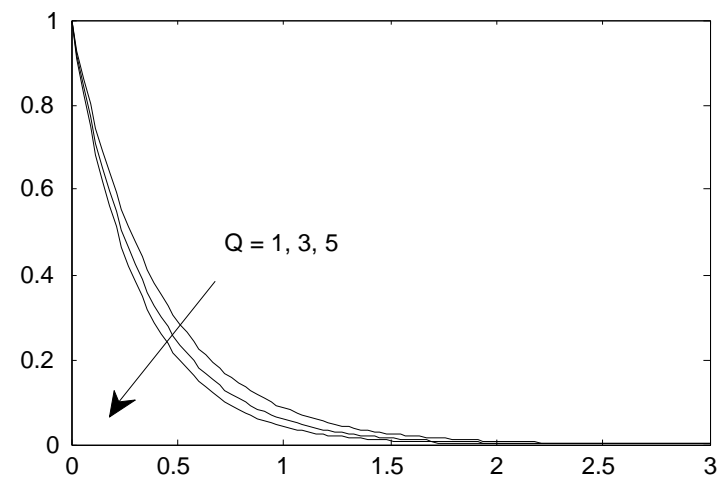

Figure 12: Temperature distribution for various values of $\mathrm{Q}$ 


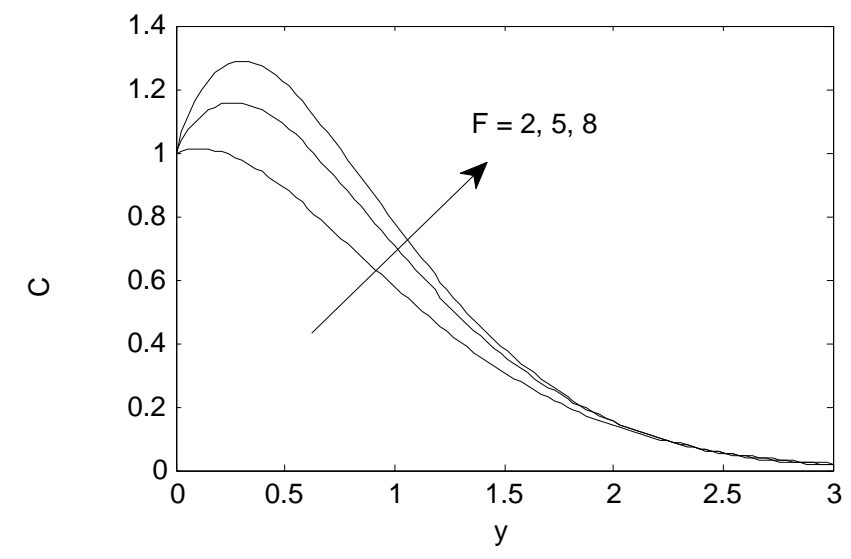

Figure 13: Concentration distribution for various values of $\mathrm{F}$

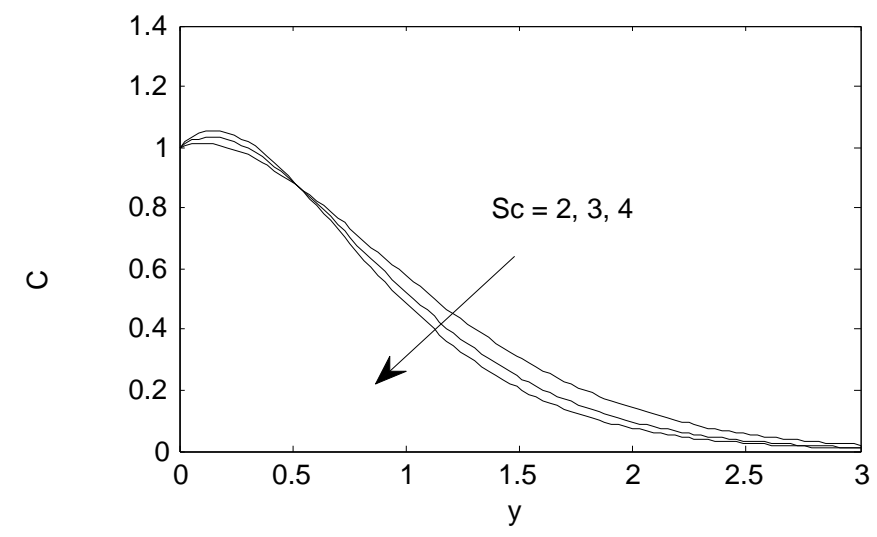

Figure 14: Concentration distribution for various values of Sc

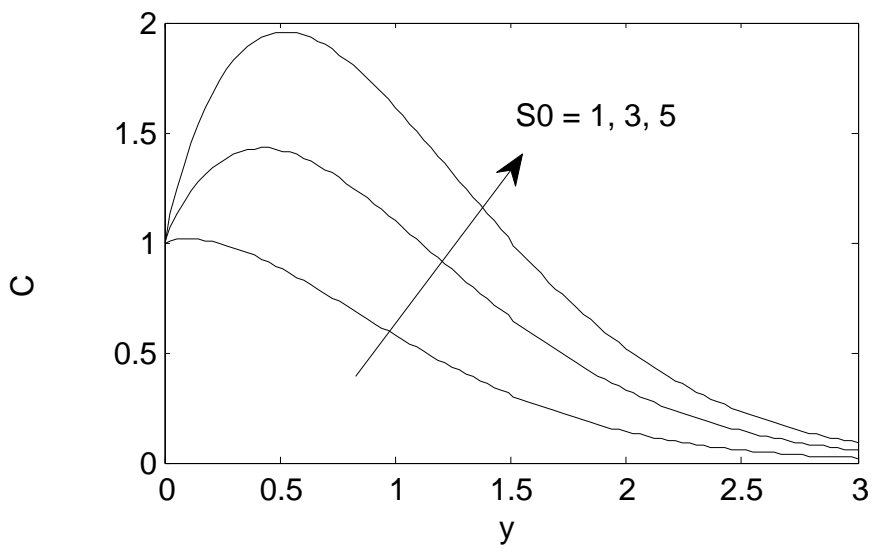

Figure 15: Concentration distribution for various values of S0 


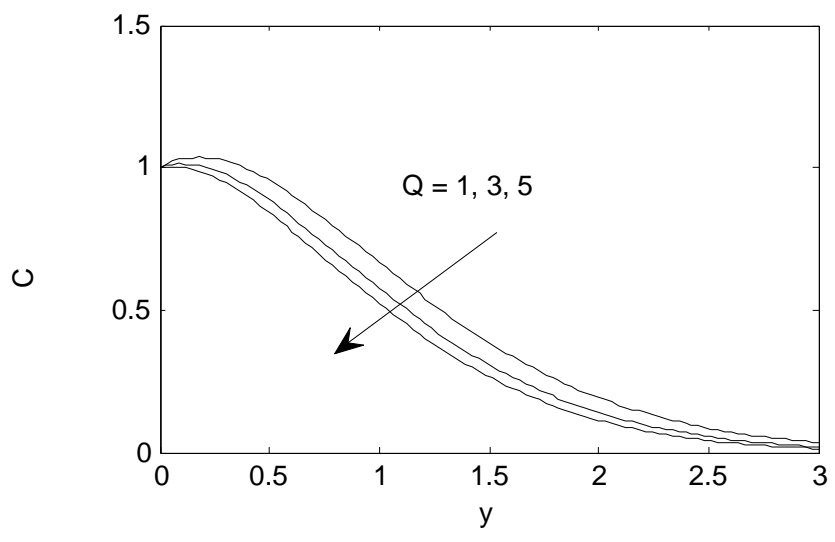

Figure 16: Concentration distribution for various values of $\mathrm{Q}$

Table (1) displays that enhancing Prandtl number Pr, radiation parameter $(F)$, Soret number $S_{0}$, Grashof number Gr, Grashof number Gm, Source parameter (Q), Hall current parameter (m) results an increasing Skin-friction coefficient. While it decreases with increase of Schmidt number Sc, magnetic field parameter M, Heat inclination angle $\alpha$. Tables (2) shows the effects of Prandtl number Pr, radiation parameter F, Heat source parameter $\mathrm{Q}$ on rate of heat transfer $(\mathrm{Nu})$. It is noticed that the rate of heat transfer increases with the increase of Pr, F and Q. Table (3) exhibits that increasing Prandtl number $\mathrm{Pr}$, Soret number $\mathrm{S}_{0}$, radiation parameter $\mathrm{F}$, Heat source parameter Q, the rate of mass transfer (Sh) increases. Enhancing Schmidt number Sc, the Sherwood number decreases.

Table 1: Skin friction

\begin{tabular}{|l|l|l|l|l|l|l|l|l|l|l|}
\hline Pr & $\mathbf{F}$ & $\mathbf{S c}$ & $\mathbf{S 0}$ & $\mathbf{M}$ & $\mathbf{G r}$ & $\mathbf{G m}$ & $\mathbf{Q}$ & $\boldsymbol{\alpha}$ & $\mathbf{m}$ & $\boldsymbol{\tau}$ \\
\hline 0.71 & 2.00 & 2.00 & 1.00 & 2.00 & 2.00 & 2.00 & 3.00 & 0.52 & 0.50 & 6.1704 \\
\hline $\mathbf{0 . 9 0}$ & 2.00 & 2.00 & 1.00 & 2.00 & 2.00 & 2.00 & 3.00 & 0.52 & 0.50 & 7.5069 \\
\hline 0.71 & $\mathbf{5 . 0 0}$ & 2.00 & 1.00 & 2.00 & 2.00 & 2.00 & 3.00 & 0.52 & 0.50 & 5.4181 \\
\hline 0.71 & 2.00 & $\mathbf{3 . 0 0}$ & 1.00 & 2.00 & 2.00 & 2.00 & 3.00 & 0.52 & 0.50 & 4.8501 \\
\hline 0.71 & 2.00 & 2.00 & $\mathbf{1 . 5 0}$ & 2.00 & 2.00 & 2.00 & 3.00 & 0.52 & 0.50 & 8.1027 \\
\hline 0.71 & 2.00 & 2.00 & 1.00 & $\mathbf{2 . 5 0}$ & 2.00 & 2.00 & 3.00 & 0.52 & 0.50 & 4.1946 \\
\hline 0.71 & 2.00 & 2.00 & 1.00 & 2.00 & $\mathbf{3 . 0 0}$ & 2.00 & 3.00 & 0.52 & 0.50 & 7.7803 \\
\hline 0.71 & 2.00 & 2.00 & 1.00 & 2.00 & 2.00 & $\mathbf{3 . 0 0}$ & 3.00 & 0.52 & 0.50 & 7.1027 \\
\hline 0.71 & 2.00 & 2.00 & 1.00 & 2.00 & 2.00 & 2.00 & $\mathbf{5 . 0 0}$ & 0.52 & 0.50 & 6.1409 \\
\hline 0.71 & 2.00 & 2.00 & 1.00 & 2.00 & 2.00 & 2.00 & 3.00 & $\mathbf{0 . 7 9}$ & 0.50 & 5.9477 \\
\hline 0.71 & 2.00 & 2.00 & 1.00 & 2.00 & 2.00 & 2.00 & 3.00 & 0.52 & $\mathbf{1 . 0 0}$ & 8.9851 \\
\hline
\end{tabular}

Table 2: Nusselt number

\begin{tabular}{|l|l|l|l|}
\hline Pr & F & $\mathbf{Q}$ & Nu \\
\hline 0.71 & 2.0 & 3.00 & 3.7622 \\
\hline $\mathbf{0 . 9}$ & 2.0 & 3.00 & 4.2336 \\
\hline 0.71 & $\mathbf{5 . 0}$ & 3.00 & 4.7784 \\
\hline 0.71 & 2.0 & $\mathbf{5 . 0 0}$ & 4.4654 \\
\hline
\end{tabular}


Table 3: Sherwood number

\begin{tabular}{|l|l|l|l|l|l|}
\hline Pr & F & Sc & S0 & Q & Sh \\
\hline 0.71 & 2.0 & 2.0 & 1.00 & 3.00 & 7.7612 \\
\hline $\mathbf{0 . 9}$ & 2.0 & 2.0 & 1.00 & 3.00 & 8.3107 \\
\hline 0.71 & $\mathbf{5 . 0}$ & 2.0 & 1.00 & 3.00 & 9.4415 \\
\hline 0.71 & 2.0 & $\mathbf{3 . 0}$ & 1.00 & 3.00 & 5.3577 \\
\hline 0.71 & 2.0 & 2.0 & $\mathbf{1 . 5 0}$ & 3.00 & 10.1371 \\
\hline 0.71 & 2.0 & 2.0 & 1.00 & $\mathbf{5 . 0 0}$ & 11.8652 \\
\hline
\end{tabular}

\section{Conclusions}

A general analytical solution for the problem of Hall current and radiation effects on MHD free convective heat and mass transfer flow past an accelerated inclined porous plate with thermal diffusion have been determined using Laplace transform technique. The expressions for Velocity, temperature, concentration, Skin friction, the rate of heat transfer in the form of Nusselt number and the rate of mass transfer in the form of Sherwood number have been derived and discussed through graphs and tables. From the study the following conclusions can be drawn:

- The velocity profile increases with increase in $\operatorname{Pr}, \mathrm{Gr}, \mathrm{Gm}, \mathrm{Q}, \mathrm{F}, \mathrm{So}$, and M while it decreases with increase in Sc, $m$ and $\alpha$.

- The temperature decreases with increase in values of Pr and F.

- The temperature decreases with increase in values of $\mathrm{Q}$.

- The Concentration increases with increase in F and So, while it decreases with increase in Sc and Q.

- Velocity on skin friction increases with increase in Pr, Gr, Gm, So, F, Q and m, while it decreases with increase in Sc, M and $\alpha$.

- The rate of heat transfer expressed in terms of Nusselt number increases with increase in Pr, F and Q.

- The rate of mass transfer expressed in terms of Sherwood number increases with increase in $\mathrm{Pr}, \mathrm{F}, \mathrm{So}$ and Q, while it decreases with increase in Sc.

\section{References}

[1] Raptis, A., Singh, A.K., "MHD free convection flow past an accelerated vertical plate". International Communications in Heat and Mass Transfer, Vol.10(4), p313-321, 1983.

[2] Duwairi, H. M., Al-Kahlawi, Y., "MHD cojugate mixed convection heat transfer over a vertical hollow cylinder embedded in a porous medium". International Journal of Heat and Technology, Vol. 24(1), p123-128, 2006.

[3] Seddeek, M.A., "The effect of variable voscosity on hydromagnetic flow and heat transfer past a contnuously moving porous boundary with radiation" Int. Commun Heat Mass, Vol. 27(7), p1037-1046, 2000.

[4] Abdelkhalek, M.M., "The skin friction in the MHD mixed convection stagnation point with mass transfer", Int. commun Heat Mass, Vol.33, 249-258, 2006.

[5] Chowdhury, M. K., Islam, M.N., MHD free convection flow of visco-elastic fluid past an infinite vertical porous plate. Heat Mass transfer 36, 439-447, 2000.

[6] Singh, P.K., Heat and Mass Transfer in MHD Boundary Layer Flow past an Inclined Plate with Viscous Dissipation in Porous Medium, International Journal of Scientific \& Engineering Research, Volume 3 (6), June-2012. 
[7] Rajesh, V. and Varma, S.V.K, Radiation and mall transfer effects on MHD free convection flow past an exponentially accelerated vertical plate with variable temperature, ARPN J. of Engg., and App. Sci., 4(6), 2009, 20-26.

[8] Takhar, H. S. and Ram, P. C., Magnetohydrodynamics free convection flow of water at $4^{\circ} \mathrm{C}$ through a porous medium, Int. Commun. Heat Mass Transfer, vol. 21, 1994, 371.

[9] Das and Das, "MHD free convection near a moving vertical plate in the presence of thermal radiation. Moldavian Journal of the Physical sciences, vol. 8 no. 3-4, 2009.

[10] Sharma P. R. and Singh G, "effects of variable thermal conductivity and heat source/sink on MHD flow near a stagnation point on a linearly stretching sheet", Journal of Applied Fluid Mechanics, vol. 2, no. 1, 2009, pp. 13-21.

[11] Chen, "Heat and mass transfer effects with variable wall temperature and concentration", Acta Mathematica, vol. 172, (2004).

[12] Pavlov K.B., Magnetohydrodynamic flow of an incompressible viscous fluid caused by the deformation of a plane surface, Magn. Gidrondin, vol.4, pp.146-152.

[13] Chakrabarti, A and Gupta AS (1979) Hydromagnetic flow of heat and mass transfer over a stretching sheet, Quart. Appl. Math 33:1979, 73-78.

[14] Andersson, H. I., MHD flow of a visco-elastic fluid past a stretching surface, Acta Mech.95: 1992, 227-230.

[15] Andersson, H.I., Bech, K.H., and Dandapat, B.S., Magnetohydrodynamic flow of a Power- law fluid over a stretching surface, Int. J. Non-Linear Mech. 27: 1992, 929-936.

[16] Alam, M.S., Rahman, M.M., Sattar, M.A. (2009), "On the effectiveness of viscous dissipation and Joule heating on steady magneto hydrodynamic heat and mass transfer flow over an inclined radiate isothermal permable surface in the presence of thermophoresis", Commun Nonlinear Sci. Numer Simul, Vol. 14, p2132-2143.

[17] G.W. Sutton, A. Sherman, Engineering Magnetohydrodynamics, McGraw-Hill, New York, 1965.

[18] H.A. Attia, Hall current effects on the velocity and temperature fields of an unsteady Hartmann flow, Can. J. Phys. 76 (9) (1998) 739.

[19] Raju, M. C., Reddy, N. A., Varma, S. V. K. , Hall-current effects on unsteady MHD flow between stretching sheet and an oscillating porous upper parallel plate with constant suction", Thermal science, Vol. 15, No.2, pp. 45-48, 2011.

[20] Ravikumar, V., Raju, M. C., Raju, G. S. S., MHD three dimensional Couette flow past a porous plate with heat transfer, IOSR Jour. Maths., Vol. 1, no.3, pp. 3-9, 2012.

[21] Raju, M. C., Varma, S.V.K ., Reddy, N. A., Radiation and mass transfer effects on a free convection flow through a porous medium bounded by a vertical surface, Journal of Future Engineering and Technology, Vol. 7, No: 2, pp. 7-12 2012.

[22] Reddy, T.S. Raju, M.C. \& Varma, S. V. K., Unsteady MHD radiative and chemically reactive free convection flow near a moving vertical plate in porous medium, JAFM, Vol.6, no.3, pp. 443451, 2013.

[23] Raju, M.C., Reddy, N.A., Varma, S. V. K., Analytical study of MHD free convictive, dissipative boundary layer flow past a porous vertical surface in the presence of thermal radiation, chemical reaction and constant suction, Ain Shams Engineering Journal, Vol. 5 (4), 2014, 1361-1369. DOI: 10.1016/j.asej.2014.07.005

[24] RaJu, M.C., Varma, S.V.K., Seshaiah, B., Heat transfer effects on a viscous dissipative fluid flow past a vertical plate in the presence of induced magnetic field, Ain Shams Engineering Journal, Vol.6, No.1, 2015, 333-339. doi:10.1016/j.asej.2014.07.009.

[25] Chandra, R.P, Raju, M.C, Raju, G.S.S., Thermal and solutal buoyancy effect on MHD boundary layer flow of a visco-elastic fluid past a porous plate with varying suction and heat source in the presence of thermal diffusion. J Appl Computat Math 4: 249, 2015. doi:10.4172/21689679.1000249 . 
[26] Raju M.C, Veeresh C, Varma S.V.K, Rushi Kumar B, Vijaya Kumar A.G., Heat and mass transfer in MHD mixed convection flow on a moving inclined porous plate. J Appl Computat Math 4: 259, 2015. doi:10.4172/2168-9679.1000259.

[27] Reddy, L.R.M., Raju, M.C., Raju, G.S.S., Convective ramped temperature and concentration boundary layer flow of a chemically reactive heat absorbing and radiating fluid over a vertical plate in conducting field with Hall current., Innov Ener Res., Vol.5(1) (2016), pp.1-20. DOI: http://dx.doi.org/10.4172/ier.1000130.

[28] Chandra P.R., M.C. Raju, G.S.S. Raju, Magnetohydrodynamic convective double diffusive laminar boundary layer flow past an accelerated vertical plate, International Journal of Engineering Research in Africa, Vol. 20 (2016), 80-92, doi:10.4028/www.scientific.net/JERA.20.80.

[29] Harikrishna, L., Veerakrishna, M., Raju, M.C., Hall current effects on unsteady MHD flow in a rotating parallel plate channel bounded by porous bed on the lower half Darcy lap wood model, Mathematical sciences International research journal, Vol.4 (2015), 29-39.

[30] Reddy, T.S., Raju, M.C., Varma, S.V.K., Hall current effects on MHD free convection flow through a porous medium bounded by a vertical surface, Proceedings of the International Conference on Mathematics in Engineering \&Business Management, March 9-10, 188-192, 2012.

[31] Reddy, P.G., Umamaheswar, M., Raju, M. C., Varma, S. V. K., Magneto-convective and radiation absorption fluid flow past an exponentially accelerated vertical porous plate with variable temperature and concentration, International Journal of Mathematics Trends and Technology (IJMTT). V31(1):26-33 March 2016.

[32] Umamaheswar, M., Varma, S. V. K., Raju, M.C., Chamkha, A.J., Unsteady MHD Free Convective Double Diffusive Visco-Elastic Fluid Flow Past an Inclined Permeable Plate in the presence of viscous dissipation and heat absorption, Special topics and Reviews in Porous media: An international Journal., Vol.6 (4). 2016, pp. 333-342.

[33] Raju, M. C., Chamkha, A. J., Philip, J., Varma, S.V.K., Soret effect due to mixed convection on unsteady magnetohydrodynamic flow past a semi-infinite vertical permeable moving plate in presence of thermal radiation, heat absorption and homogenous chemical reaction, International Journal of Applied and Computational Mathematics. DOI 10.1007/s40819-016-0147-x.

[34] Philip, J., Raju, M. C., Chamkha, A. J. and Varma, S.V. K., "MHD Rotating Heat and Mass Transfer Free Convective Flow Past an Exponentially Accelerated Isothermal Plate with Fluctuating Mass Diffusion." International Journal of Industrial Mathematics, Vol. 6, No. 4, 2014 Article ID IJIM-00478, 10 pages, 297-306.

[35] Chamkha, A. J., Reddy, T. S., Raju, M. C., and Varma, S.V.K., "Unsteady MHD Free Convection Flow Past an Exponentially Accelerated Vertical Plate with Mass Transfer, Chemical Reaction and Thermal Radiation." International Journal of Microscale and Nanoscale Thermal and Fluid Transport Phenomena, Vol.5, no.1, 2014, pp. 57-75.

[36] Umamaheswar, M., Varma, S. V. K., Raju, M. C., Numerical study of Magneto-Convective and radiation absorption fluid flow past an exponentially accelerated vertical porous plate with variable temperature and concentration in the presence of Soret and Dufour effects, IOSR Journal of Mathematics, Volume 12, Issue 2 Ver. I (Mar. -Apr.2016), PP 109-120. DOI: 10.9790/57281221109120.

[37] Rao, B. M., Reddy, G. V., Raju, M. C., Varma, S. V. K., MHD transient free convection and chemically reactive flow past a porous vertical plate with radiation and temperature gradient dependent heat source in slip flow regime, IOSR Journal of Applied Physics, Vol. 3(6), 2013, 22 32.

*Corresponding author.

E-mail address: sivaiahgunti@gmail.com 